\title{
Análisis de los factores influyentes en la prevalencia de las úlceras de pie en pacientes en hemodiálisis
}

\author{
María Jesús Lechuga Domínguez, Ana Rodríguez García, Eva María Vázquez Caridad \\ Unidad de hemodiálisis. Hospital San Rafael. A Coruña. España
}

\section{Resumen}

Introducción: Los pacientes en tratamiento renal sustitutivo con terapia de hemodiálisis, tienen elevado riesgo de sufrir úlceras en los pies, debido a la presencia de numerosos factores de riesgo asociados.

Objetivos: El presente estudio, se plantea como objetivo principal establecer la prevalencia de úlceras en los pies, en los pacientes que realizan tratamiento con hemodiálisis en el Hospital San Rafael de A Coruña.

Como objetivo secundario, valorar los factores de riesgo de ulceración del pie.

Material y Método: Se realiza un estudio descriptivo transversal, en el que incluyen a los 131 pacientes con insuficiencia renal crónica (IRC) en tratamiento con hemodiálisis, que cumplen los criterios de inclusión. Se ha marcado como variable principal del estudio la presencia o ausencia de úlcera en el pie.

Se valora el estado vascular, con la palpación de pulsos y con registro de sonido doppler de las arterias pedia y tibial posterior, además se realiza el cálculo del índice tobillo brazo (ITB). Se valora el estado neurológico, tras la exploración de la sensibilidad protectora con monofilamento y de la sensibilidad profunda con diapasón graduado.

Resultados: El 10,7\% de los pacientes del estudio tienen úlcera activa en el pie. No se logra palpar pulso en la arteria pedia en un $43,5 \%$ de los pacientes y en la arteria tibial posterior en un $60 \%$ de la muestra estudiada. El $25 \%$ de los pacientes presenta un valor de ITB, por debajo de 0,90 y un $13,5 \%$ presenta arterias no compresibles.

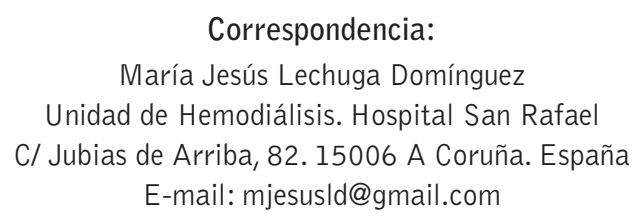

Presentan alteración de sensibilidad profunda un $48,9 \%$ de los pacientes y alteración de la sensibilidad protectora un $44,3 \%$.

Conclusión: La prevención primaria a través de protocolos estandarizados de exploración del pie en las unidades de hemodiálisis, podrá minimizar la posibilidad de ulceraciones que comprometan la calidad de vida de los pacientes.

Sistemáticamente habrá que realizar una valoración vascular, neurológica y de alteraciones podológicas, en la que se incluyan tanto a los pacientes con diabetes mellitus, como a los pacientes sin diabetes.

PALABRAS CLAVE: hemodiálisis; úlcera; pie; prevalencia; prevención.

\section{Analysis of influential factors in the prevalence of foot ulcers in hemodialysis patients}

\begin{abstract}
Introduction: Patients on renal replacement therapy with haemodialysis therapy are at high risk of suffering foot ulcer. This is due to the large number of associated risk factors.
\end{abstract}

Objective: The main goal of this study is to determine the prevalence of foot ulcers in patients who are being treated with haemodialysis therapy at San Rafael Hospital, A Coruña.

The secondary aim is to analyze the risk factors associated to foot ulcers. 
Methods: A cross-sectional descriptive study is performed, including 131 patients suffering from chronic kidney disease. All these patients meet the inclusion criteria. Presence or absence of foot ulcers has been considered the main variable in this paper.

A vascular examination is performed following the procedure of inspect, palpate and registration of doppler sound of pedis and posterior tibial artery. Furthermore, an ankle-brachial index (ABI) is calculated.

A neurological examination is also performed. A monofilament is used to test protective sensation while a tuning fork is used to test deep sensitivity.

Results: $10,7 \%$ of the patients studied suffer from active ulcer on the foot. We unsuccessfully attempted to palpate a pulse in the pedis artery in $43,5 \%$ of patients. The attempt to palpate a pulse in the posterior tibial artery was also unsuccessful in $60 \%$ of patients. $25 \%$ of patients were found an anklebrachial index (ABI) less than 0,90 and a $13,5 \%$ has noncompressible arteries.

$49,9 \%$ of the patients under study show alteration of deep sensation and a $44,3 \%$ show alteration of protective sensation.

Conclusion: The primary prevention through standardized protocols of the foot examination in the haemodialysis units will be able to reduce the chances of ulceration that can endanger patients' quality of life.

A vascular, neurological and podiatric disorders examination must be done systematically, including patients with and without diabetes mellitus.

KEYWORDS: haemodialysis; ulcer; foot; prevalence; prevention.

\section{Introducción}

Los pacientes con insuficiencia renal crónica (IRC) en estadio 5D que reciben tratamiento con hemodiálisis (HD) presentan numerosos factores de riesgo de ulceración en el pie ${ }^{1,2,3}$, comparables a los existentes en la población diabética ${ }^{4}$. Al-Thani $\mathrm{H}$ et al., apuntan que los pacientes a tratamiento con HD presentan un alto índice de enfermedad arterial periférica, de ulceraciones y de amputaciones en los pies 5 . Por su parte $\mathrm{Ndip} \mathrm{A}$ et al., Ilegaron a la conclusión de que la HD por sí misma, podría llegar a considerarse un factor de riesgo independiente de aparición de úlceras en los pies en pacientes diabéticos ${ }^{6}$. Ante la coexistencia de diabetes mellitus e IRC-5D el peligro de ulceración y amputación de la extremidad inferior es más elevado que cuando se presentan estos factores de manera aislada ${ }^{4,7,8}$.

Orimoto et al., realizaron un estudio retrospectivo en el que valoraron desde el año 1980 hasta el año 2011 la supervivencia de pacientes a tratamiento con HD con lesiones en los pies, así como la viabilidad del miembro inferior. Los autores exponen entre sus conclusiones que los pacientes en tratamiento con HD con lesiones en los pies presentan un mal pronóstico y unas elevadas tasas de amputación y de mortalidad?.

La vigilancia e identificación de úlceras en los pies se incluye desde el año 2007 en el informe de la U.K. Renal Registry como predictor de mortalidad en la población que recibe tratamiento con $\mathrm{HD}^{3}$.

La prevalencia de factores de riesgo de úlceras en los pies en pacientes diabéticos con IRC-5D en tratamiento con HD está bien documentada ${ }^{6,7,10-14}$, pero no ocurre lo mismo con la población con IRC-5D no diabética.

En el estudio realizado por Al- Thani et al. ${ }^{5}$, se encontraron que de una cohorte de 252 pacientes en tratamiento con HD, el $17 \%$ presentaba úlceras en los pies. De los 152 pacientes que recibían tratamiento con HD y que además presentaban diabetes mellitus, un $23 \%$ tenía úlceras en los pies. De los 100 pacientes en tratamiento con HD que no presentaban diabetes mellitus un $17 \%$ tenía úlceras en los pies.

No es habitual, dentro de las rutinas de las unidades de diálisis, encontrarnos con programas de salud dirigidos especialmente al cuidado y vigilancia de los pies ${ }^{15}$. En algunos centros, estos se limitan a folletos informativos y a consejos dirigidos tan solo a la población diabéti$\mathrm{ca}^{1}$. Un adecuado programa de supervisión de la salud 
podológica, podría reducir el número y gravedad de las amputaciones de las extremidades inferiores de los pacientes que reciben tratamiento con $\mathrm{HD}^{16}$.

A pesar de los datos referenciados, no existen demasiados estudios llevados a cabo en España que relacionen la aparición de úlceras en los pies de los pacientes con IRC en tratamiento con HD. En el año 2009, Eloísa Bernal Pedreño et al., realizaron exploraciones del pie a los pacientes diabéticos de una unidad de $\mathrm{HD}^{17}$. Azucena García Viejo, llevó a cabo en 2012, un estudio de la enfermedad arterial periférica y pie diabético en pacientes en programa de $\mathrm{HD}^{12}$. Es por ello, que podría resultar de interés la obtención de datos epidemiológicos que justifiquen una posible intervención preventiva o terapéutica desde el punto de vista podológico en la población a estudio.

La American Diabetes Association en su publicación standards of medical care in diabetes- $2015^{18}$, recomienda basándose en la evidencia (grado de recomendación B) realizar un enfoque multidisciplinario hacia los pacientes con úlceras del pie y/o los pacientes con pies de alto riesgo, como por ejemplo los pacientes de diálisis.

La exploración física, es una forma rápida, sencilla y eficaz tanto para la valoración vascular como neurológica del pie ${ }^{19}$. En la valoración vascular debe incluirse la palpación de pulsos, para poder identificar la presencia de isquemia. Debe incluir también la valoración de la presión arterial tomada con doppler a nivel maleolar y el cálculo del ITB ${ }^{20}$. En la valoración neurológica, pruebas como el estudio de percepción con el monofilamento de Semmes-Weinstein y de percepción de la vibración con el diapasón graduado de Rydel-Seiffer, nos ayudan a descartar la presencia de neuropatía ${ }^{20}$.

La Conferencia Nacional de Consenso sobre Úlceras de la Extremidad Inferior (CONUEI) ${ }^{21}$, las define como:

- "Lesión en la extremidad inferior, espontánea o accidental, cuya etiología pueda referirse a un proceso patológico sistémico o de la extremidad y que no cicatriza en el intervalo temporal esperado."

La American Diabetes Association (ADA) en su publicación standards of medical care in diabetes- 2015, establece que el riesgo de ulceración y amputación en el pie está aumentado en los pacientes que presentan los siguientes factores de riesgo ${ }^{18}$ :
- Historia previa de amputación

- Historia previa de ulceración

- Neuropatía periférica

- Deformidades podológicas

- Enfermedad vascular periférica

- Incapacidad visual

- Nefropatía diabética (especialmente pacientes en diálisis)

- Mal control glucémico

- Hábito tabáquico

Aunque el estadio terminal de la IRC está asociado con el desarrollo de úlceras en los pies y consecuentemente con la amputación de la extremidad inferior, la evidencia sobre cómo contribuyen los factores de riesgo para el desarrollo de las complicaciones a nivel del pie, es limitada ${ }^{4}$.

\section{Objetivos}

Establecer la prevalencia de úlceras en los pies de una muestra de pacientes con IRC en tratamiento con HD en nuestra unidad.

Relacionar el estado vascular y neurológico en los pies de pacientes con IRC en tratamiento con HD, con la presencia de úlceras.

Determinar la relación entre padecer diabetes y la presencia de úlceras y alteraciones de la sensibilidad en los pies.

Relacionar la presencia de úlceras en los pies con el tiempo que el paciente lleva en HD.

Establecer la relación entre sexo, edad, Índice Masa Corporal (IMC) e índice de Charlson con la presencia de úlceras en los pies.

\section{Material y Método}

El estudio se inició con una búsqueda bibliográfica de la información más relevante sobre el tema. Parte de las referencias bibliográficas se obtuvieron tras la búsqueda manual en las obras de texto disponibles y la otra parte se obtuvo tras la búsqueda informática en el repositorio de documentos en acceso abierto PubMed, en las bases de datos médicas Medline, Web of Science, Dialnet, Scopus y a través del buscador Google Académico. 
Se llevó a cabo un estudio descriptivo transversal al que se incorporaron todos los pacientes que cumplieron con los criterios de inclusión y que recibían tratamiento renal sustitutivo (TRS) en la unidad de hemodiálisis (HD) del "Hospital San Rafael de A Coruña".

En el momento del estudio se dializaban en nuestra unidad un total de 154 pacientes. Estimando que la prevalencia de úlceras en los pies en pacientes a tratamiento con HD se sitúe alrededor de $17 \% \%^{5}$ y queriendo tener una precisión del 8,4\% en la estimación de la prevalencia con una confianza del $95 \%$, se precisan estudiar al menos 90 sujetos.

Se diseñó una hoja de recogida de datos (anexo I), en la que se incluyeron tanto los datos obtenidos durante la exploración como los datos de antecedentes médicos de los individuos a estudio.

Doppler: Valoración vascular. Se utilizó un detector bidireccional de bolsillo para la búsqueda de sonido doppler en las arterias tibial posterior y pedia. Se registra como presente 0 ausente. Además se utilizó para la determinación de la presión arterial sistólica (PAs) en las arterias braquial y tibial posterior.

Esfigmomanómetro: Valoración vascular. Se utilizó un tensiómetro manual, para la determinación de la PAs en miembros superiores (MMSS), miembros inferiores (MMII).

Índice tobillo brazo ${ }^{22}$ : Valoración vascular. EI ITB es una técnica no invasiva, efectiva para el diagnóstico y valoración de la isquemia en MMII. Se obtiene del cociente ente la PAs en el tobillo y la PAs en el brazo. Los valores se interpretarán como ${ }^{(22)}$ :

- ITB $>1,3$ arteria no compresible.

- ITB 1,00- 1,29 Normal.

- ITB 0,91-0,99 Valores límite.

- ITB 0,41- 0,90 Isquemia leve/moderada.

- ITB $<0,41$ Isquemia severa.

Una de las mayores limitaciones del ITB, en pacientes en HD y en pacientes diabéticos, se presenta cuando encontramos vasos no compresibles. Esta situación se asocia a una importante calcificación de la arteria, que origina una presión arterial falsamente elevada en las arterias del tobillo ${ }^{23}$. A pesar de esto, se recomienda la determinación del ITB para realizar el cribado de los pacientes.
Monofilamento Semmes-Weinstein ${ }^{24}$ : Valoración de la sensibilidad protectora. Se utilizó el monofilamento de calibre $n=5,07$. Filamento de nailon unido habitualmente a un mango de plástico, que al doblarse aplica una presión constante sobre la piel de $10 \mathrm{gr}$. Se ejerce presión perpendicularmente sobre la superficie cutánea hasta que el hilo se incurve, momento en el que se valora la percepción. Se aplica en intervalos de tres segundos y se realizan dos aplicaciones en cada una de las zonas a valorar. Se determinaron 5 puntos de exploración $\left(1^{\mathrm{a}}\right.$, $3^{\mathrm{a}}$ y $5^{\mathrm{a}}$ cabeza metatarsal, zona distal de la cara plantar del hallux, cara dorsal del $1^{\circ}$ espacio interdigital) en cada uno de los pies. Se evitaron zonas con hiperqueratosis y/o úlcera ${ }^{25}$. Se considera que existe pérdida de la sensación protectora, cuando el paciente es incapaz de detectar la presión en una o más de las zonas anatómicas exploradas ${ }^{25}$.

Diapasón graduado de Rydel-Seiffer ${ }^{24}$ : Valoración de la sensibilidad profunda. Se utilizó un diapasón graduado, que dispone de dos ramas con ocho puntos de desplazamiento. Con el movimiento de vibración impreso se interpretan los triángulos que se forman en las ramas. Se sujeta el diapasón por el mango y se percuten sus ramas con la palma de la mano contraria. Se aplica su base en la zona a explorar. Se valoraron tres zonas anatómicas, articulación interfalángica (AIF) del hallux, maléolo tibial y maléolo peroneal. El paciente debe indicar el momento en el que deja de percibir la vibración. Momento en el que se realiza la lectura del triángulo formado en la sordina. En cada una de las zonas se realizarán tres lecturas de la sordina del diapasón. Se considera que existe riesgo de ulceración cuando la lectura del triángulo es $\leq 4^{24}$ en uno 0 ambos pies.

Índice de comorbilidad de Charlson: Evalúa la esperanza de vida a los diez años, en función de la edad y de 19 items que influyen de forma concreta en la esperanza de vida del sujeto. En general se considera ausencia de comorbilidad 0-1 puntos, comorbilidad baja 2 puntos y comorbilidad alta $\geq 3$ puntos $^{26}$.

Índice de masa corporal ${ }^{27}$ : EI IMC nos ofrece datos para la valoración del estado nutricional del paciente. Se obtiene al dividir el peso y su talla ${ }^{2}$. Los valores se interpretarán como ${ }^{27}$ :

- IMC < 18,5 Peso Insuficiente

- IMC 18,5-24,9 Peso Normal

- IMC 25- 26,9 Sobrepeso Grado I

- IMC 27-29,9 Sobrepeso Grado II

- IMC 30-34,9 Obesidad Grado I 
- IMC 35- 39,9 Obesidad Grado II

- IMC 40-49,5 Obesidad Grado III (mórbida)

- IMC >50 Obesidad Grado IV (extrema)

Alteraciones podológicas ${ }^{1,2,3,4}$ : Se valoró durante la exploración clínica y se registró la presencia de deformidades, como son el hallux valgus y deformidades digitales. Así mismo, valoramos la presencia de alteraciones dermatológicas, como son las hiperqueratosis, helomas, fisuras en el talón, maceración y/o fisuras interdigitales. También registramos la presencia de alteraciones ungueales, como variaciones estructurales, de color, patología traumática o patología infecciosa. Con la presencia de uno o más de estos cambios, se consideró que el paciente tiene alteraciones podológicas.

\section{Análisis Estadístico}

Se utilizó el software para análisis de datos SPSS. Se realizó un análisis descriptivo de las variables incluidas en el estudio. Las cualitativas se presentan con sus valores absolutos y porcentajes con los correspondientes intervalos de confianza al 95\% (IC 95\%). De las variables cuantitativas se describirá la media, mediana, desviación típica y cuartiles.

Para la comparación de variables cualitativas se realizó un test de Chi cuadrado, mientras que para la comparación de medias se realizó una $t$ de student 0 test de Mann Whitney, según proceda, tras determinar la normalidad con el test de Kolmogorov Smirnov.

Finalmente, se comprobó la relación de la existencia de úlceras con distintas variables. Para ello se realizó un análisis multivariante de regresión logística, en el que como variable dependiente se consideró la presencia de úlceras y como variables independientes todas aquellas variables que en el análisis univariante hayan mostrado una relación estadísticamente significativa con la presencia de úlceras $(p<0,10)$.

\section{Resultados}

Se examinaron un total de 131 pacientes, entre los que hay 85 varones $(64,9 \%)$ y 46 mujeres $(35,1 \%)$. Con una edad media de 69,4 años, siendo la edad mínima de 20 años y la máxima de 90 . El $75 \%$ de los pacientes estudiados tiene más de 63 años.

La media de tiempo que llevan en HD es de 40,2 meses. El paciente con menos tiempo lleva Imes y el paciente con más tiempo lleva 235 meses.

En relación al IMC la media es de 26,99 sobrepeso grado I, con una desviación típica de 5,03. La media del índice de Charlson es de 7,67, siendo el mínimo de 2 y el máximo de 14. El índice de comorbilidad medio en pacientes en HD es alto.

La prevalencia de otros factores como el hábito tabáquico es del $11,5 \%$ y de hábito alcohólico es del $2,3 \%$.

En la tabla 1, se muestra el análisis de las variables cuantitativas.

Tabla 1. Tabla de variables cuantitativas.

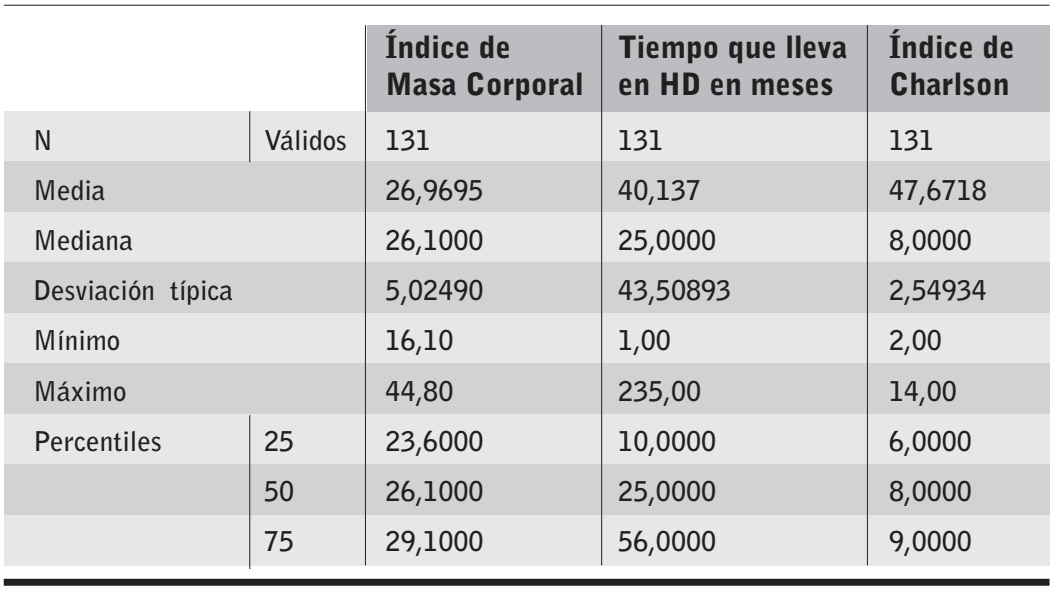

La prevalencia de úlcera en el pie es del 10,7\%. De los pacientes examinados 14 tienen algún tipo de úlcera activa. Presentan antecedentes de úlcera un $13,7 \%$ (18 pacientes) y se observa amputación en un $5,3 \%$ de la muestra, ( 7 pacientes).

Un $37.4 \%$ de los pacientes son diabéticos (49 pacientes), de los cuales, un $20,4 \%$ son diabéticos tipo I y un $79,6 \%$ son diabéticos tipo II.

El porcentaje de pacientes diagnosticados de arteriopatía periférica es de un 17,6\%, (23 pacientes). Con diagnóstico previo de angioplastia y/o by-pass hay un $5,3 \%$, (7 pacientes) y muestran síntomas vasculares un $16 \%$, (21 pacientes).

Para valorar el estado vascular en los pies, se realizó palpación manual de los pulsos, en las arterias 
tibial posterior y pedia. En un $60 \%$ de los pacientes, no es palpable el pulso en la arteria tibial posterior (78 pacientes) y en un $43,5 \%$ no es palpable el pulso en la arteria pedia (57 pacientes).

Además se realizó la búsqueda de sonido doppler en las citadas arterias del pie. Se aprecia sonido doppler en la arteria tibial posterior en el $97,7 \%$ de los pacientes (127), y en la arteria pedia en el $98,5 \%$ (128). Si bien estos porcentajes son elevados, cabe destacar que tan solo se documenta la presencia o ausencia de sonido doppler. No se profundiza en la valoración del tono, volumen, ni amplitud del sonido. Tampoco se analiza de forma exhaustiva el tipo de onda que se percibe.

Se completa la valoración vascular, con la determinación del ITB. Del total de la muestra, se pudo determinar el ITB en 111 pacientes. La media de los resultados se encuentra dentro de los límites de la normalidad, siendo 1,04, con una desviación típica de 0,30 . El $26,1 \%$ de los pacientes, tiene una valor de ITB por debajo de 0,90 , asociado a isquemia leve/moderada, el 2,7\% presenta un ITB por debajo de 0,41, asociado a isquemia severa.

Presentan arterias no compresibles, con valor de ITB por encima de 1,30 , un $13,5 \%$. No se pudo realizar la compresión completa de la arteria, por lo tanto no se pudo determinar el valor del ITB en un 15,3\% de los pacientes.

Para valorar el estado neurológico en los pies de los pacientes, se estudian la sensibilidad profunda y la sensibilidad protectora. Un $48,9 \%$ de los pacientes, presentó alteración de la sensibilidad profunda (64 pacientes), medida con diapasón graduado de Rydel-Sheiffer. Un $44,3 \%$ de los pacientes presentó alteración de la sensibilidad protectora (58 pacientes), medida con filamento de Semmes-Weinstetin.

Como se puede observar en la tabla 2 , un $72,4 \%$ de los pacientes de nuestra unidad que presentan alteración de la sensibilidad pro-
Tabla 3. Tabla de contingencia Diabetes* Úlcera activa* Alteración sensibilidad profunda* Alteración sensibilidad protectora.

\begin{tabular}{|c|c|c|c|c|c|c|c|c|}
\hline & & & \multicolumn{2}{|c|}{ Úlcera activa } & \multicolumn{2}{|c|}{$\begin{array}{l}\text { Alteración } \\
\text { Sensibilidad } \\
\text { Profunda }\end{array}$} & \multicolumn{2}{|c|}{$\begin{array}{l}\text { Alteración } \\
\text { Sensibilidad } \\
\text { Protectora }\end{array}$} \\
\hline & & & No & Sí & No & Sí & No & Si \\
\hline \multirow[t]{4}{*}{ Diabetes } & \multirow[t]{2}{*}{ No } & Recuento & 77 & 5 & \multirow[b]{2}{*}{$59,8 \%$} & 33 & 54 & 28 \\
\hline & & $\begin{array}{l}\text { \% dentro } \\
\text { de Diabetes }\end{array}$ & $93,9 \%$ & $6,1 \%$ & & $40,2 \%$ & $65,9 \%$ & $34,1 \%$ \\
\hline & \multirow[t]{2}{*}{ Sí } & Recuento & 40 & 9 & \multirow{2}{*}{$\begin{array}{l}18 \\
36,7 \%\end{array}$} & \multirow{2}{*}{$\begin{array}{l}31 \\
63,3 \%\end{array}$} & \multirow{2}{*}{$\begin{array}{l}19 \\
38,8 \%\end{array}$} & \multirow{2}{*}{$\begin{array}{l}30 \\
61,2 \%\end{array}$} \\
\hline & & $\begin{array}{l}\text { \% dentro } \\
\text { de Diabetes }\end{array}$ & $81,6 \%$ & $18,4 \%$ & & & & \\
\hline
\end{tabular}

tectora, presentan además alteración de la sensibilidad profunda.

En cuanto a los resultados de alteraciones podológicas. De los pacientes explorados, un $60,3 \%$ presentaban deformidades en los pies (79 pacientes), un $68,7 \%$ presentaban alteraciones dermatológicas $(90$ pacientes) y un $74 \%$ alteraciones ungueales (97 pacientes).

\section{Relación entre las variables estudiadas}

Los pacientes diabéticos de la muestra, tienen 3,5 veces más riesgo de tener úlcera en el pie que los no diabéticos, IC $95 \%=1,1-11$ (18,4\%, versus $6,1 \%$; $p=0,02$ ). Por otra parte, tienen 2,6 veces más riesgo de presentar alteraciones de la sensibilidad profunda, IC $95 \%=1,2-5,3(63,3 \%$ versus $40,2 \% ; p=0,01)$. Tienen además 3 veces más riesgo de tener alteración de la sensibilidad protectora IC 95\% = 1,5- 6,3 $(61,2 \%$ versus $34,1 \% ; p=0,003)$. Podemos observar la tabla 3 de contingencia de diabetes y úlcera previa, alteración de la sensibilidad profunda y alteración de la sensibilidad protectora.

\begin{tabular}{|c|c|c|c|c|}
\hline & & & \multicolumn{2}{|c|}{$\begin{array}{c}\text { Alteración Sensibilidad } \\
\text { Profunda }\end{array}$} \\
\hline & & & No & Sí \\
\hline \multirow[t]{4}{*}{$\begin{array}{l}\text { Alteración Sensibilidad } \\
\text { Protectora }\end{array}$} & No & Recuento & 51 & 22 \\
\hline & & $\begin{array}{l}\% \text { dentro de Alteración } \\
\text { Sensibilidad Protectora }\end{array}$ & $69,9 \%$ & $30,1 \%$ \\
\hline & Sí & Recuento & 16 & 42 \\
\hline & & $\begin{array}{l}\text { \% dentro de Alteración } \\
\text { Sensibilidad Protectora }\end{array}$ & $27,6 \%$ & $72,4 \%$ \\
\hline
\end{tabular}


No se encuentra relación estadísticamente significativa entre la presencia úlcera activa, con el tiempo que el paciente lleva en HD. La media de meses en pacientes con úlcera activa es de 46,4 y en pacientes sin úlcera es de 39,4 meses. Tampoco se encontró relación entre la presencia de úlcera activa con IMC ( $p=$ $0,95)$, ni con el Índice de Charlson $(p=0,42)$. Aplicando la prueba de Mann-Whitney se encontró relación entre la edad y la presencia de úlcera activa. En la muestra estudiada, las úlceras se presentan en pacientes con menos edad $(61,6$ versus 71,3 años de media; $p=0,01$ )

Se encontró mayor prevalencia de úlcera activa en hombres $12,9 \%$ frente a un $6,5 \%$ en mujeres, aunque este dato no es significativo $p=0.26$.

Los pacientes con úlcera activa presentan ITB menor que los pacientes sin úlcera. $(0,79$ versus 1,06 valor medio de ITB; $p=0,02$ ).

Con respecto a la valoración neurológica, no hay diferencia significativa entre los pacientes con úlcera activa y alteración de la sensibilidad profunda $(64,3 \%$ versus $47 \% ; p=0,22$ ). Sin embargo si existe relación entre la presencia de úlcera activa y la alteración de la sensibilidad protectora $(71,4 \%$ versus $41 \% ; p=0,03)$. Se puede observar la tabla 4 de contingencia de úlcera activa y alteración de la sensibilidad profunda y protectora.

Tabla 4. Tabla de contingencia úlcera Activa* Alteración sensibilidad profunda* Alteración sensibilidad protectora.

\begin{tabular}{|c|c|c|c|c|c|c|}
\hline & & & \multicolumn{2}{|c|}{$\begin{array}{l}\text { Alteración } \\
\text { Sensibilidad } \\
\text { Profunda }\end{array}$} & \multicolumn{2}{|c|}{$\begin{array}{l}\text { Alteración } \\
\text { Sensibilidad } \\
\text { Protectora }\end{array}$} \\
\hline & & & No & Sí & No & $\mathbf{S i}$ \\
\hline \multirow[t]{4}{*}{ Úlcera activa } & \multirow[t]{2}{*}{ No } & Recuento & 62 & 55 & 69 & 48 \\
\hline & & $\begin{array}{l}\text { \% dentro } \\
\text { de Úlcera activa }\end{array}$ & $53,0 \%$ & $47,0 \%$ & $59,0 \%$ & $41,0 \%$ \\
\hline & \multirow[t]{2}{*}{ Sí } & Recuento & 5 & 9 & \multirow[b]{2}{*}{2060} & 10 \\
\hline & & $\begin{array}{l}\text { \% dentro } \\
\text { de Úlcera activa }\end{array}$ & $35,7 \%$ & $64,3 \%$ & & $71,4 \%$ \\
\hline
\end{tabular}
versus $2,4 \%$ ).

\section{Discusión} dado de los pies. hemodiálisis.

estadísticamente significativa entre la presencia de alteraciones dermatológicas y úlcera activa ( $14,4 \%$

Cada semana o cada mes, según pauta de cada enfermera referente, en nuestra unidad, realizábamos una exploración visual, de los pies de nuestros pacientes con diabetes. Detectamos algunas lesiones, cuya evolución fue satisfactoria en algunos casos y más tórpida en otros, llegando hasta la pérdida del miembro afectado, en varias ocasiones.

Esta exploración, se basaba en la realización un examen visual de los pies. Se buscaba la presencia de heridas o lesiones y ofrecíamos consejos sobre el cui-

Percibimos que nuestros pacientes en HD, presentaban numerosos problemas, relacionados con su salud podológica y nos planteamos realizar una exploración más exhaustiva, en la que incluiríamos tanto a los pacientes diabéticos como a los pacientes no diabéticos.

Los resultados del estudio vienen a confirmarnos la elevada prevalencia de factores de riesgo de ulceración en el pie, en nuestra población de pacientes en

Freeman et al. ${ }^{2}$, investigaron la frecuencia de estos factores de riesgo en sujetos con IRC. EI porcentaje de pacientes diabéticos en su estudio con úlcera activa, fue del $16 \%$, en nuestra unidad, este porcentaje aumenta hasta el $18,4 \%$. En cuanto a pacientes en HD sin diabetes, en su muestra, ninguno presentaba úlcera activa. En el estudio observacional de cinco años de AlThani et al. ${ }^{5}$, el porcentaje de pacientes diabéticos con úlcera activa aumenta hasta un $23 \%$. Sin embargo, el porcentaje de

En cuanto a las alteraciones podológicas, los pacientes con deformidades en los pies presentan 1,2 veces más posibilidades de presentar úlcera, IC $95 \%=1,1$ $1,3(17,7 \%$ versus $0 \% ; p=0,001)$. Los pacientes con alteraciones ungueales, presentan 1,2 veces más posibilidades de presentar úlcera, IC $95 \%=1,1-1,3$ $(14,4 \%$ versus $0 \% ; p=0,02)$. No sé encontró relación pacientes en HD con úlcera sin diabetes es del $7 \%$, dato que se asemeja más al 6,1\% obtenido en nuestro estudio.

Para determinar la presencia de neuropatía, se exploraron alteraciones de sensibilidad profunda y protectora. En el estudio de Kaminski et al. ${ }^{4}$, un 25,4\% de 
los pacientes presentaba neuropatía periférica. Nia $\mathrm{J}$ Jones et al. ${ }^{3}$, en su unidad un $23 \%$ de los pacientes, presenta alteración de la sensibilidad protectora y un $25 \%$ presenta alteración de la sensibilidad profunda. En nuestra unidad este porcentaje aumenta hasta el $44,3 \%$ y un $48,9 \%$ respectivamente.

Refiriéndonos a la valoración vascular, citamos el estudio de Cusolito et al. ${ }^{1}$. En más del $50 \%$ de sus pacientes, no fue palpable el pulso en la arteria tibial posterior. Este porcentaje disminuye hasta el 30\%, si nos referimos a la arteria pedia. En nuestra unidad estos porcentajes aumentan hasta un $60 \%$ y $43,5 \%$ respectivamente.

Al igual que el estudio de Cusolito.$^{1}$, se ha encontrado diferencia entre la palpación del pulso de forma manual, y la presencia del flujo arterial con doppler. Siendo este último más sensible para la valoración. En un $97,7 \%$ de los pacientes se detectó flujo arterial con doppler en la arteria tibial posterior y en un $98,5 \%$ en la arteria pedia.

EI ITB nos muestra la presencia de arteriopatía en un $26,1 \%$ de nuestros pacientes. En el estudio de Azucena García ${ }^{12}$, su porcentaje es del $35,3 \%$, en pacientes diabéticos. La media de ITB en nuestros pacientes diabéticos es de 0,94 , resultado dentro de los valores límite de la normalidad, pero muy cercano a la isquemia leve/moderada ${ }^{22}$. Detectamos que el ITB medio de los pacientes con úlcera activa, se encuentra dentro de los límites de isquemia leve/moderada 0,79.

Aunque una de las limitaciones en el cálculo del ITB en pacientes en HD está asociada a las calcificaciones arteriales distales, aun así, se considera una herramienta efectiva y útil para el diagnóstico de enfermedad vascular periférica, en pacientes con y sin diabetes ${ }^{28}$.

Un $79 \%$ de los pacientes del estudio de $\mathrm{Nia}$ Jones $^{3}$ tiene patología podológica, en ella engloba, la presencia de dedos en garra, hallux valgus, cabezas metatarsales prominentes, helomas y patología de uñas. Kaminski et al. ${ }^{4}$, diferencia entre deformidad en el pie, un $69 \%$ y patología cutánea y ungueal, un $81,7 \%$. En nuestra muestra distinguimos deformidades del pie, presentes en un $60,3 \%$ de los pacientes, alteraciones dermatológicas, en un $68,7 \%$ y alteraciones ungueales, en un $74 \%$.

Es interesante que el tiempo que el paciente lleva en $H D$, no es un factor de riesgo significativo, en el riesgo de ulceración en el pie. En el meta-análisis elaborado por Kaminski et al., en el año $2015^{29}$, relaciona esta situación, con un sesgo de supervivencia. Los pacientes con lesiones en los pies de causa isquémica, presentan muerte prematura por causas vasculares y no realizan largos periodos de tiempo en HD.

\section{Conclusiones}

A la vista de los resultados obtenidos, podemos decir, que como esperábamos la presencia de factores de riesgo de ulceración en el pie, en pacientes diabéticos, es elevado. De igual manera encontramos que la población no diabética en HD, también se ve afectada por la presencia de estos mismos factores, lo que nos orienta hacia una prevención estándar en las unidades de tratamiento.

Se observa una elevada prevalencia de sintomatología silente (alteraciones sensibilidad, ausencia y/o debilidad de pulsos, alteraciones de ITB...) en pacientes en HD que podría derivar en una ulcera de pie.

Deberían incorporarse por tanto, para su uso sistematizado, protocolos de exploración y seguimiento del pie (a nivel vascular, sensitivo y de alteraciones morfológicas/ funcionales) en todos los pacientes en tratamiento renal sustitutivo con HD.

Mediante la realización de exploraciones no invasivas, que se pueden llevar a cabo durante el tratamiento por el personal de enfermería, podemos valorar los factores de riesgo presentes en cada paciente. Instaurar los cuidados oportunos puede minimizar la posibilidad de este tipo de lesiones.

\section{Agradecimientos:}

Este trabajo ha sido realizado gracias a la concesión de la beca "Jandry Lorenzo" 2016, patrocinada por la Sociedad Española de Enfermería Nefrológica SEDEN.

Recibido: 25 agosto 2016

Revisado: 30 septiembre 2016

Modificado: 21 febrero 2017

Aceptado: 3 marzo 2017 


\section{Bibliografía}

1. Locking-Cusolito H, Harwood L, Wilson B, Burgess $\mathrm{K}$, Elliot M, Gallo $\mathrm{K}$, et al. Prevalence of risk factors predisposing to foot problems in patients on hemodialysis. Nephrol Nurs J 2005 JulAug;32(4):373-384.

2. Freeman A, May K, Frescos N, Wraight PR. Frequency of risk factors for foot ulceration in individuals with chronic kidney disease. Intern Med J 2008;38(5):314-320.

3. Jones N. Prevalence of risk factors for foot ulceration in a general haemodialysis population. International wound journal 2013;10(6):683.

4. Kaminski M. Prevalence of risk factors for foot ulceration in patients with end-stage renal disease on haemodialysis. Intern Med J 2012;42(6):e120.

5. Al-Thani H, El-Menyar A, Koshy V, Hussein A, Sharaf $A$, Asim M, et al. Implications of foot ulceration in hemodialysis patients: a 5-year observational study. J Diabetes Res 2014;2014:945075.

6. Ndip A, Rutter MK, Vileikyte L, Vardhan A, Asari A, Jameel $M$, et al. Dialysis treatment is an independent risk factor for foot ulceration in patients with diabetes and stage 4 or 5 chronic kidney disease. Diabetes Care 2010 Aug;33(8):1811-1816.

7. Ndip A, Lavery LA, Lafontaine J, Rutter MK, Vardhan $A$, Vileikyte $L$, et al. High levels of foot ulceration and amputation risk in a multiracial cohort of diabetic patients on dialysis therapy. Diabetes Care 2010 Apr;33(4):878-880.

8. Korzets $A$, Ori $Y$, Rathaus $M$, Plotnik N, Baytner $S$, Gafter $U$, et al. Lower extremity amputations in chronically dialysed patients: a 10 year study. Isr Med Assoc J 2003 Jul;5(7):501-505.

9. Orimoto $Y$, Ohta $T$, Ishibashi $H$, Sugimoto I, Iwata $H$, Yamada $T$, et al. The prognosis of patients on hemodialysis with foot lesions. J Vasc Surg 2013 Nov;58(5):1291-1299.

10. Benabdellah N, Karimi I, Bentata Y, Haddiya I. Approach to podiatric status of the diabetic patient in chronic hemodialysis in a Moroccan hospital. Pan Afr Med J 2013 Sep 12;16:13.

11. Ndip A, Lavery LA, Boulton AJ. Diabetic foot disease in people with advanced nephropathy and those on renal dialysis. Current diabetes reports 2010;10(4):283-290.
12. García Viejo A. Enfermedad arterial periférica y pié diabético en pacientes en programa de hemodiálisis. Enfermería Nefrológica 2012;15(4):255-264.

13. Batista-García $F$, Hernández $M$, Suria $S$, Esparza N, Checa MD. Diabetic foot and renal failure. Theoretical and practical considerations. Nefrología 2012 May 14;32(3):399.

14. Schomig M, Ritz E, Standl E, Allenberg J. The diabetic foot in the dialyzed patient. J Am Soc Nephrol 2000 Jun;11(6):1153-1159.

15. $0^{\prime}$ Hare A, Johansen K. Lower-extremity peripheral arterial disease among patients with endstage renal disease. J Am Soc Nephrol 2001 Dec;12(12):2838-2847.

16. Wilson B. Implementation of a foot assessment program in a regional satellite hemodialysis setting. CANNT journal 2013;23(2):41.

17. Bernal Pedreño E, Salces Sáez E, Sambruno Giráldez A. Exploración del pié a los pacientes diabéticos de una unidad de hemodiálisis. Revista de la Sociedad Española de Enfermería Nefrológica 2009;12(2):35-40.

18. American Diabetes Association. Standards of medical care in diabetes-2015. Diabetes Care 2015 Jan;38 Suppl 1:1-94.

19. Viadé Juliá J. Pie diabético: guía para la práctica clínica. $2^{a}$ ed. Madrid: Médica Panamericana; 2013.

20. Viadé Julià J. Pie diabético: guía práctica para la prevención, evaluación y tratamiento. Madrid: Médica Panamericana; 2006.

21. Verdú J, Marinel-lo J, Armans E, Carreño P, March J, Martín V, et al. Documento de Consenso CONUEI. Conferencia Nacional de Consenso sobre úlceras de la Extremidad Inferior-CONUEI. 2009.

22. March-García J, Fontcuberta-García J, BenitoFernández L, Martí-Meste F, Vila-Coll R. Guía básica para el estudio no invasivo de la isquemia crónica de miembros inferiores. Angiología 2009;61(Suppl. 1):S75-S92.

23. Guindo J, Martínez-Ruiz MD, Gusi G, Punti $\mathrm{J}$, Bermúdez $P$, Martínez-Rubio A. Métodos diagnósticos de la enfermedad arterial periférica. Importancia del índice tobillo-brazo como técnica de criba. Revista española de cardiología suplementos 2009;9(4):11-17. 
24. Marinel·lo Roura J. Úlceras De La Extremidad Inferior. $2^{\mathrm{a}}$ ed. Barcelona: Glosa; 2011.

25. Boulton AJ, Armstrong DG, Albert SF, Frykberg RG, Hellman R, Kirkman MS, et al. Comprehensive foot examination and risk assessment: a report of the task force of the foot care interest group of the American Diabetes Association, with endorsement by the American Association of Clinical Endocrinologists. Diabetes Care 2008 Aug;31(8):16791685.

26. Gil-Bona J, Sabaté A, Bovadilla JMM, Adroer R, Koo M, Jaurrieta E. Valor de los índices de CharIson y la escala de riesgo quirúrgico en el análisis de la mortalidad operatoria. Cirugía Española 2010;88(3):174-179.
27. Martínez JA, Portillo MdP. Fundamentos de nutrición y dietética: bases metodológicas y aplicaciones. Madrid: Panamericana; 2011.

28. Ng SY, Cheng SW, Chu WL, Lui SL, Lo WK. Screening by trained nurses for peripheral vascular disease in continuous ambulatory peritoneal dialysis patients with and without diabetes. Perit Dial Int 2003 Dec;23 Suppl 2:S134-8.

29. Kaminski MR, Raspovic $A$, McMahon LP, Strippoli GF, Palmer SC, Ruospo M, et al. Risk factors for foot ulceration and lower extremity amputation in adults with end-stage renal disease on dialysis: a systematic review and meta-analysis. Nephrol Dial Transplant 2015 0ct;30(10):1747-1766.

\section{Anexo I}

\section{Hoja de Registro de Datos}

\begin{tabular}{|c|c|c|}
\hline Sexo & Mujer & Edad _ Años \\
\cline { 2 - 2 } & Hombre & \\
\hline
\end{tabular}

\begin{tabular}{|llllll}
\hline Peso & _kg & Talla & $\mathrm{cm}$ & IMC \\
\hline
\end{tabular}

\begin{tabular}{|c|c|c|}
\hline \multicolumn{3}{|l|}{ ANTECEDENTES } \\
\hline Úlcera activa & Si & No \\
\hline Tiempo desde inicio en hemodiálisis & & Meses \\
\hline Diagnóstico de diabetes mellitus & $\mathrm{Si}$ & No \\
\hline Tipo de diabetes & Tipo 1 & Tipo 2 \\
\hline Diagnóstico de arteriopatía periférica & $\mathrm{Si}$ & No \\
\hline Historia de angioplastia y/o by-pass & $\mathrm{Si}$ & No \\
\hline Síntomas Vasculares & Si & No \\
\hline Úlcera previa & $\mathrm{Si}$ & No \\
\hline Amputación & Si & No \\
\hline Hábito tabáquico & Si & No \\
\hline Hábito alcohólico & Si & No \\
\hline \multicolumn{3}{|l|}{ Índice comorbilidad de Charlson } \\
\hline \multicolumn{3}{|l|}{ ALTERACIONES PODOLÓGICAS } \\
\hline & Pie Derecho & Pie Izquierdo \\
\hline $\begin{array}{l}\text { Deformidades } \\
\text { (Hallux valgus, deformidad digital) }\end{array}$ & Si No & Si No \\
\hline $\begin{array}{l}\text { Alteraciones dermatológicas } \\
\text { (Hiperqueratosis, heloma, fisura) }\end{array}$ & Si No & Si No \\
\hline $\begin{array}{l}\text { Alteraciones ungueales } \\
\text { (Estructurales, color, traumática, infecciosa) }\end{array}$ & Si No & Si No \\
\hline
\end{tabular}




\begin{tabular}{|c|c|c|c|c|}
\hline \multicolumn{5}{|c|}{ VALORACIÓN NEUROLÓGICA } \\
\hline \multirow[b]{2}{*}{ Diapasón } & \multicolumn{2}{|c|}{ Pie Derecho } & \multicolumn{2}{|c|}{ Pie Izquierdo } \\
\hline & $0-4$ & $5-8$ & $0-4$ & $5-8$ \\
\hline \multicolumn{5}{|l|}{$1^{\text {a }}$ AIF } \\
\hline \multicolumn{5}{|c|}{ Maleolo interno } \\
\hline \multicolumn{5}{|c|}{ Maleolo externo } \\
\hline $\begin{array}{l}\text { Alteración } \\
\text { Sensibilidad } \\
\text { Profunda }\end{array}$ & Si & No & Si & No \\
\hline \multicolumn{5}{|c|}{$\begin{array}{l}\text { Monofilamento } \\
\text { Leyenda: } \\
\text { Normal (+) } \\
\text { Alterada (-) }\end{array}$} \\
\hline $\begin{array}{l}\text { Alteración } \\
\text { Sensibilidad } \\
\text { Protectora }\end{array}$ & Si & No & Si & No \\
\hline
\end{tabular}

\begin{tabular}{|c|c|c|c|c|}
\hline \multicolumn{5}{|c|}{ VALORACIÓN VASCULAR } \\
\hline \multirow[b]{2}{*}{ Pulso (manual) } & \multicolumn{2}{|c|}{ Pie Derecho } & \multicolumn{2}{|c|}{ Pie Izquierdo } \\
\hline & Presente & Ausente & Presente & Ausente \\
\hline \multicolumn{5}{|l|}{ Tibial Posterior } \\
\hline \multicolumn{5}{|l|}{ Pedio } \\
\hline Doppler & Audible & No Audible & Audible & No Audible \\
\hline \multicolumn{5}{|l|}{ Tibial Posterior } \\
\hline \multicolumn{5}{|l|}{ Pedio } \\
\hline & \multicolumn{2}{|c|}{ Derecha } & \multicolumn{2}{|c|}{ Izquierda } \\
\hline \multicolumn{5}{|l|}{ PAs Tobillo } \\
\hline \multicolumn{5}{|l|}{ PAs Brazo } \\
\hline Valor ITB & & & & \\
\hline
\end{tabular}

